\title{
Adaptive Fuzzy Sliding Mode and Robust Tracking Control for Manipulators with Uncertain Dynamics
}

\author{
Sanxiu Wang (iD \\ College of Electronic and Information Engineering, Taizhou University, Taizhou, Zhejiang 318000, China \\ Correspondence should be addressed to Sanxiu Wang; wsx8188@163.com
}

Received 19 May 2020; Revised 25 June 2020; Accepted 7 July 2020; Published 25 July 2020

Guest Editor: Qiang Chen

Copyright (c) 2020 Sanxiu Wang. This is an open access article distributed under the Creative Commons Attribution License, which permits unrestricted use, distribution, and reproduction in any medium, provided the original work is properly cited.

\begin{abstract}
In response to the issue of the trajectory tracking control problem of manipulators with uncertain parameters and external disturbance, an adaptive fuzzy sliding mode robust control algorithm is proposed. Sliding mode control (SMC) is adopted to perform robotic manipulator trajectory tracking control. Then, a fuzzy logic system is used for adaptive adjustment of switching gain of the SMC and to reduce the buffeting problem. Next, compensation is made by using the robust controller in consideration of the impacts of unmodeled dynamics and external disturbance. The simulation experiment on a two axes robotic manipulator shows that, with the proposed control method, the sliding mode control input signal is kept smooth, and the manipulator has high trajectory tracking precision.
\end{abstract}

\section{Introduction}

The multijoint robotic manipulator system is strongly coupled, time-variant, and nonlinear. In the recent years, the high-precision control of a manipulator has attracted wide attention from the academia and the industrial circle. A series of achievements have been made in the research on the trajectory tracking of the manipulator's joint space. Control algorithms such as sliding mode control [1-3], adaptive control [4-6], robust control [7, 8], and iterative learning control [9] have been proposed. Among them, the sliding mode control algorithm is simple and highly robust to parameter changes and disturbance, making it especially suitable for high-precision tracking control of nonlinear systems such as a robotic manipulator. However, high-frequency buffeting exists in SMC, and the vibration degree is affected by the switching gain. Normally, in order to ensure system stability, ample switching gain is selected, which aggravates the buffeting in sliding mode control. Such buffeting may cause the existence of unmodeled high-frequency components in the system or even cause system instability.

To overcome the chattering problem of sliding mode control, various methods have been proposed in the literature, among which the well-known method is to apply a saturation function to the SMC control gain when the sliding surface is located in the boundary of the sliding hyperplane $[10,11]$. This method is simple; however, it cannot guarantee the output convergence. That is to say, there is a nonzero steadystate error in the output. Another common method is to use a disturbance observer to estimate and compensate the external disturbance and uncertainty, so as to solve the chattering problem of sliding mode control $[12,13]$. However, it has high accuracy only for constant or slow changing disturbances. Another way to solve the chattering problem of sliding mode control is to apply fuzzy logic control. In the compact set, fuzzy control can approach any continuous function due to its characteristic of universal approximation and does not depend on the system model $[14,15]$. So, it has gained wide applications in robot adaptive control. Thus, in this paper, a new adaptive fuzzy SMC algorithm is proposed for robotic manipulators. The adoption of the fuzzy logic system in adjusting the switching gain of SMC in an adaptive way can effectively eliminate the buffeting of the sliding mode control algorithm. At the same time, considering that uncertain parameters, unmodeled dynamics, and external disturbances will reduce the control performance of the system [16-18], a robust controller is used to compensate these uncertainties and achieve accurate tracking of the manipulator. 
Additionally, most industrial robotic manipulators complete tasks within the task space through terminal actuators, and their expected trajectory is also described using the task space. So, to implement joint space control, it is necessary to convert the task space coordinate of the robotic manipulator into the joint space. Then, through high-performance joint space tracking control algorithm, the high precision tracking control of the manipulator is achieved.

The organization of the paper is as follows. The model descriptions are given in Section 2, including conversion between the task space coordinate and joint space angle position and manipulator dynamics model. The design of the control algorithm is detailed in Section 3, and an adaptive fuzzy sliding mode controller and robust controller are constructed. Simulation results are presented in Section 4. Finally, conclusions constitute Section 5.

\section{Model Descriptions}

Trajectory tracking in the task space refers to the tracking of the the desired trajectory by the end effector. However, as the actuators of most manipulators are installed on the manipulator body and their joints, the tracking of the trajectory of the manipulator within the joint space is to track the given expected joint angles of the manipulators one by one. Thus, in order to achieve control in joint space, the trajectory of the task space should be firstly converted into that of joint space.

2.1. Conversion between the Task Space Coordinate and Joint Space Angle. The movement coordinate at the terminal of the robotic manipulator in the task space $(x, y)$ is converted into the two-joint angle position $\left(q_{1}, q_{2}\right)$ [19].

According to Figure 1, the following formulas can be obtained:

$$
\left\{\begin{array}{l}
x=l_{1} \cos q_{1}+l_{2} \cos \left(q_{1}+q_{2}\right) \\
y=l_{1} \sin q_{1}+l_{2} \sin \left(q_{1}+q_{2}\right)
\end{array}\right.
$$

Adding the square of $x$ and that of $y$, we get

$$
x^{2}+y^{2}=l_{1}^{2}+l_{2}^{2}+2 l_{1} l_{2} \cos q_{2} .
$$

Thus, the following formula can be obtained:

$$
q_{2}=\cos ^{-1}\left(\frac{x^{2}+y^{2}-l_{1}^{2}-l_{2}^{2}}{2 l_{1} l_{2}}\right) .
$$

Take $p_{1}=\arctan (y / x)(x \geq 0)$ or $p_{1}=\pi+\arctan (y / x)$ $(x<0), p_{2}=\arccos \left(x^{2}+y^{2}+l_{1}^{2}-l_{2}^{2} /\left(2 l_{1} \sqrt{x^{2}+y^{2}}\right)\right)$, then

$$
q_{1}= \begin{cases}p_{1}-p_{2}, & q_{2}>0, \\ p_{1}+p_{2}, & q_{2} \leq 0 .\end{cases}
$$

2.2. Manipulator Dynamics Model. A robotic manipulator is a kind of complex MIMO control system, with the characteristics of being nonlinear and time-varying. Its dynamic equation is described as follows [20]:

$$
M(q) \ddot{q}+C(q, \dot{q}) \dot{q}+G(q)=\tau+d .
$$

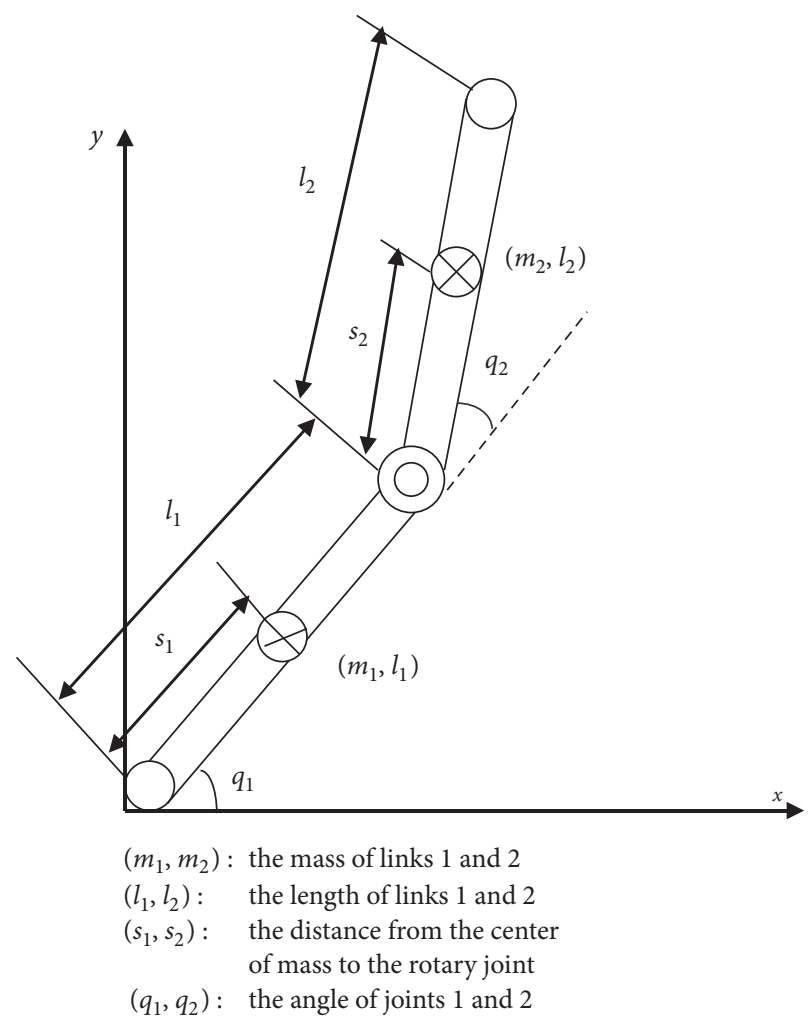

Figure 1: Two-link robotic manipulator.

Here, $q$ means the joint position and $\dot{q}, \ddot{q}$ mean joint velocities and accelerations, respectively; inertia matrix $M(q) \in R^{n \times n}$, which is symmetric positive definite; $C(q, \dot{q}) \in R^{n \times n}$ means the centrifugal and Coriolis force matrix; and $G(q) \in R^{n \times 1}$ means the gravity matrix. $d \in R^{n}$ means the external disturbance and $\tau \in R^{n}$ means the control torque vector or control input of each joint.

The manipulator dynamics model has the following dynamics characteristics [20,21]:

Characteristic 1: the symmetric positive definite torque $M(q)$ is consistent and bounded for all values of $q$. For existing positive numbers $\kappa_{m}$ and $\kappa_{M}$, that satisfies the following formula:

$$
0<\kappa_{m} I \leq M(q)<\kappa_{M} I .
$$

Characteristic 2: $\forall v \in R^{n}$, and the skew symmetric matrix $M(q)-2 C(q, \dot{q})$ meets the following relationships:

$$
v^{T}(M(q)-2 C(q, \dot{q})) v=0 .
$$

Characteristic 3: for all $q \in R^{n}$, the $G(q)$ is always bounded.

\section{Control Algorithm Design}

In this part, a control algorithm containing SMC, adaptive fuzzy control, and robust control is designed for robot trajectory tracking. The control structure of the proposed method is shown in Figure 2. 


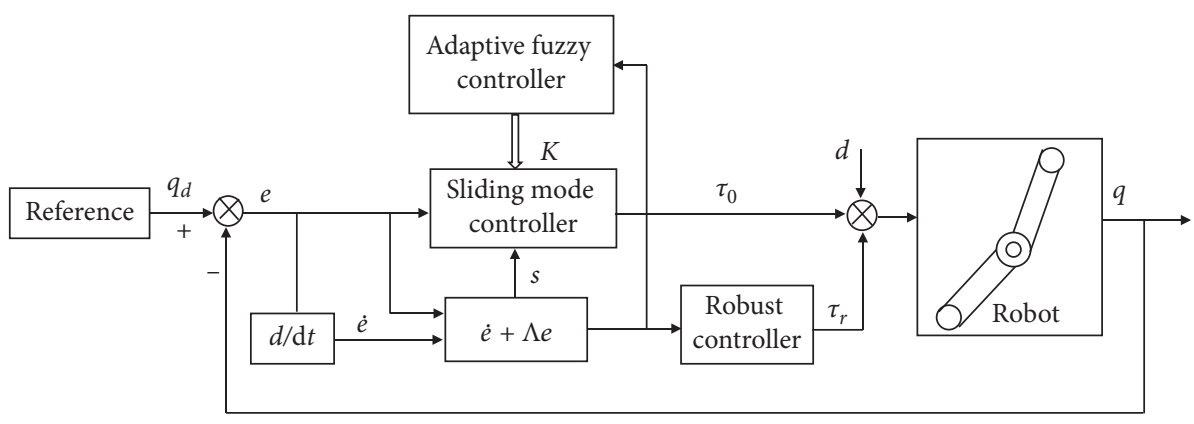

Figure 2: Diagram of the proposed sliding mode robust control system.

3.1. Design and Analysis of the Self-Adaptive Fuzzy SMC. In the actual robot system, parameter perturbation is inevitable. When the parameters of the object cannot be obtained accurately, we use $\widehat{M}(q)$ to estimate the inertia matrix $M(q) ; \widehat{C}(q, \dot{q})$ is used to estimate the centrifugal and Coriolis force matrix $C(q, \dot{q})$; and $\widehat{G}(q)$ is used to estimate the gravity matrix $G(q)$.

Let

$$
\begin{aligned}
M(q) & =\widehat{M}(q)+\Delta M(q), \\
C(q, \dot{q}) & =\widehat{C}(q, \dot{q})+\Delta C(q, \dot{q}), \\
G(q) & =\widehat{G}(q)+\Delta G(q),
\end{aligned}
$$

where $\Delta M(q)$ means estimation error of the inertia matrix, $\Delta C(q, \dot{q})$ means estimation error of the Coriolis force matrix, $\Delta G(q)$ means estimation error of the gravity matrix.

Then, define the sliding function as follows:

$$
s=\dot{e}+\Lambda e .
$$

Here, tracking error $e=q_{d}-q . q_{d}$ means expected trajectory of the joint; $q$ means actual tracking trajectory; and $\Lambda$ means a diagonal matrix with the positive definite constant.

Choosing the auxiliary signal $\dot{q}_{r}$ and $\ddot{q}_{r}$ is described as follows:

$$
\begin{aligned}
& \dot{q}_{r}=\dot{q}-s=\dot{q}_{d}-\Lambda e, \\
& \ddot{q}_{r}=\ddot{q}-\dot{s}=\ddot{q}_{d}-\Lambda \dot{e},
\end{aligned}
$$

where $\dot{q}_{d}$ and $\ddot{q}_{d}$ mean expected joint speed and accelerations, respectively.

According to the theory of sliding mode control, the controller is designed as follows:

$$
\tau_{0}=\widehat{M}(q) \ddot{q}_{r}+\widehat{C}(q, \dot{q}) \dot{q}_{r}+\widehat{G}(q)-A s-K \operatorname{sgn}(s) .
$$

Here, $K$ and $A$ are positive definite matrixes.

By combining formulas (10) and (11) and substituting formula (12) into the robot dynamic equation (5), we obtain the following relationship:

$$
\begin{aligned}
& M(q) \ddot{q}+C(q, \dot{q}) \dot{q}+G(q) \\
& \quad=M(q)\left(\ddot{q}_{r}+\dot{s}\right)+C(q, \dot{q})\left(\dot{q}_{r}+s\right)+G(q), \\
& \quad=\hat{M}(q) \ddot{q}_{r}+\widehat{C}(q, \dot{q}) \dot{q}_{r}+\widehat{G}(q)-A s-K \operatorname{sgn}(s)+d .
\end{aligned}
$$

Based on formula (8), the following formula can be obtained:

$$
\begin{aligned}
M(q) \dot{s}+(C(q, \dot{q})+A) s= & \Delta M(q) \ddot{q}_{r}+\Delta C(q, \dot{q}) \dot{q}_{r} \\
& +\Delta G(q)+d-K \operatorname{sgn}(s) \\
= & \Delta f-K \operatorname{sgn}(s),
\end{aligned}
$$

where $\quad \Delta f=\Delta M(q) \ddot{q}_{r}+\Delta C(q, \dot{q}) \dot{q}_{r}+\Delta G(q)+d$ means unmodeled terms and disturbance terms.

We choosing the Lyapunov function as follows:

$$
V=\frac{1}{2} s^{T} M(q) s
$$

Taking the derivative of the expression and substituting formula (7) into the result, we get

$$
\dot{V}=s^{T} M(q) \dot{s}+\frac{1}{2} s^{T} \dot{M}(q) s .
$$

Substituting formula (13) into the abovementioned expression, we get

$$
\begin{aligned}
\dot{V} & =s^{T} M(q) \dot{s}+\frac{1}{2} s^{T} \dot{M}(q) s \\
& =s^{T}(-(C(q, \dot{q})+A) s+\Delta f-K \operatorname{sgn}(s)+C(q, \dot{q}) s), \\
& =s^{T}(-A s+\Delta f-K \operatorname{sgn}(s)) \\
& =s^{T}(\Delta f-K \operatorname{sgn}(s))-s^{T} A s .
\end{aligned}
$$

Suppose that $\Delta f$ is bounded and satisfies the relationship $\|\Delta f\| \leq K$; then,

$$
\dot{V}=s^{T}(\Delta f-K \operatorname{sgn}(s))-s^{T} A s \leq-s^{T} A s \leq 0 .
$$

So, the system is stable.

Although the sliding mode controller is simple and effective, buffeting constitutes the main problem to be solved in the sliding control law. Also, the switching gain $K$ is the main reason for the buffeting problem. The larger the value of $K$ is, the more obvious the buffeting will appear. $K$ is used to compensate for the impact of external disturbance. For sufficient compensation, an enough large value of switching gain is required, which further aggravates the buffeting of the system. 
In order to solve the buffeting problem caused by the SMC with fixed gain, a self-adaptive fuzzy scheme is added to the conventional SMC. The fuzzy controller is used for adjustment of the switching gain in an adaptive manner so that the switching gain of SMC can be adjusted with time so as to improve the buffeting problem.

Based on the product reasoning method and the central average antifuzzy controller, the fuzzy control system is designed for adaptive adjustment of switching gain $K$ of the SMC. Let $K=\left[k_{1}, \ldots, k_{i}, \ldots, k_{n}\right]^{T}$, where $k_{i}$ denotes the fuzzy system's $i$ th output.

The output of the fuzzy system is described as follows:

$$
y(x)=\frac{\sum_{l=1}^{m} \bar{y}^{l}\left(\prod_{i=1}^{n} \mu F_{i}^{l}\left(x_{i}\right)\right)}{\sum_{l=1}^{m}\left(\prod_{i=1}^{n} \mu F_{i}^{l_{i}}\left(x_{i}\right)\right)}=\xi^{T}(x) \theta,
$$

where $\theta=\left[y^{1}, \ldots, y^{m}\right]^{T}$ means the parameter vector, $\xi(x)=\left[\xi_{1}(x), \ldots, \xi_{m}(x)\right]^{T}$ means the regression vector, and $m$ means the number of fuzzy rules.

Define

$$
\xi_{l}(x)=\frac{\prod_{i=1}^{n} \mu F_{i}^{l}\left(x_{i}\right)}{\sum_{l=1}^{m}\left(\prod_{i=1}^{n} \mu F_{i}^{l}\left(x_{i}\right)\right)} .
$$

As can be seen from formula (18), in order to satisfy $\dot{V} \leq 0, \quad$ let $\quad s^{T} K \geq 0, \quad s=\left[s_{1}, \ldots, s_{i}, \ldots, s_{n}\right]^{T}, \quad$ and $s^{T} \Delta f-s^{T} K \operatorname{sgn}(s) \leq 0$. Then, $s_{i}$ and $k_{i}$ should take the same positive and negative symbols, and $\left|s_{i}\right|,\left|k_{i}\right|$ shall change by following the identical trend.

For the fuzzy system, we choose $s_{i}$ and switching gain $k_{i}$ as the input and output, whose value is described by using five variables. The fuzzy inference rule is shown as Table 1 .

The membership function used to represent the fuzzy set is chosen as follows:

$$
\mu_{F}\left(x_{i}\right)=\exp \left[-\left(\frac{x_{i}-\alpha}{\sigma}\right)^{2}\right] .
$$

The $i$ th output of the fuzzy system is expressed as follows:

$$
k_{i}=\frac{\sum_{l=1}^{m} \bar{y}^{l} \mu F^{l}\left(s_{i}\right)}{\sum_{l=1}^{m} \mu F^{l}\left(s_{i}\right)}=\xi_{k_{i}}^{T}\left(s_{i}\right) \theta_{k_{i}} .
$$

Taking $k_{i}==\xi_{k_{i}}^{T}\left(s_{i}\right) \theta_{k_{i}}$ as the approximated value of $\Delta f_{i}$ and in view of the universal approximation theorem, there exists $\omega_{i}>0$ to satisfy the following:

$$
\left|\Delta f_{i}-\xi_{k_{i}}^{T}\left(s_{i}\right) \theta_{k_{i}}\right| \leq \omega_{i}
$$

The adaptive law is selected as follows:

$$
\dot{\tilde{\theta}}_{k_{i}}=s_{i} \xi_{k_{i}}\left(s_{i}\right) \text {. }
$$

Then, based on the switching gain adjusted by the adaptive fuzzy control, the SMC is expressed as follows:

$$
\tau_{0}=\widehat{M}(q) \ddot{q}_{r}+\widehat{C}(q, \dot{q}) \dot{q}_{r}+\widehat{G}(q)-A s-\widehat{K} .
$$

3.2. Adaptive Robust Controller Design. In this part, for unmodeled dynamics and external disturbances $d$, a robust control item is designed to eliminate their effects.
TABLE 1: Fuzzy inference rule.

$s_{i} \quad \mathrm{PM}$ PS ZO NS NM PM/NM: positive/negative medium $k_{i}$ PM PS ZO NS NM PS/NS: positive/negative small ZO: zero

The robust controller is designed as follows:

$$
\tau_{r}=-\frac{\beta^{2}}{\beta\|s\|+\varepsilon} s,
$$

where $\varepsilon>0$ means a tiny constant and $\beta$ means the upper limit for disturbance and uncertainties, which satisfy the following:

$$
\|\Delta f\| \leq \beta=\rho \mu,
$$

where $\rho=\max \left(1,\|e\|,\|e\|^{2}\right)$ is a coefficient vector; $\mu$ is an uncertainty term of the system, and its value adopts the following adaptive algorithm for auto adjustment:

$$
\dot{\tilde{\mu}}=\dot{\tilde{\mu}}=-\gamma \rho\|s\|,
$$

where $\gamma$ means a matrix with a positive definite constant and meeting the following relationships: $\widetilde{\mu}=\widehat{\mu}-\mu, \widetilde{\beta}=\widehat{\beta}-\beta$, and $\widehat{\beta}=\rho \widehat{\mu}, \widetilde{\beta}=\rho \widetilde{\mu}$.

Thus, the adaptive robust controller $\tau_{r}$ can be further described as follows:

$$
\tau_{r}=-\frac{(\rho \widehat{\mu})^{2}}{(\rho \widehat{\mu})\|s\|+\varepsilon} s .
$$

In summary, the overall control scheme is as follows:

$$
\tau=\tau_{0}+\tau_{r}
$$

\section{Simulation Analysis}

For the sake of demonstrating the validity and superiority of the control algorithm designed in this paper, simulation analysis is carried on the two-link manipulator. The specific expression for its dynamic equation is as follows:

$$
\left[\begin{array}{ll}
M_{11} & M_{12} \\
M_{21} & M_{22}
\end{array}\right] \ddot{q}+\left[\begin{array}{cc}
C_{11} & C_{12} \\
C_{21} & 0
\end{array}\right] \dot{q}+\left[\begin{array}{l}
G_{1} \\
G_{2}
\end{array}\right]=\tau+d
$$

where $\quad M_{11}=m_{1} s_{1}^{2}+m_{2}\left(l_{1}^{2}+s_{2}^{2}+2 l_{1} s_{2} \cos q_{2}\right)+I_{1}+I_{2}$, $M_{12}=M_{21}=m_{2}\left(s_{2}^{2}+l_{1} s_{2} \cos q_{2}\right)+I_{2}, \quad M_{22}=m_{2} s_{2}^{2}+I_{2}$, $C_{11}=-2 m_{2} l_{1} s_{2} \dot{q}_{2} \sin q_{2}, \quad C_{21}=-C_{12}=m_{2} l_{1} s_{2} \sin q_{2}$, and $G_{1}=2 m_{2} l_{1} s_{2} g \cos \left(q_{1}+q_{2}\right)+\left(m_{1} s_{1}^{2}+m_{2} l_{1}^{2}+m_{1} l_{1} s_{1}-m_{1} l_{1}^{2}\right) g$ $\cos \left(q_{1}\right), G_{2}=2 m_{2} l_{1} s_{2} g \cos \left(q_{1}+q_{2}\right)$.

The simulation parameters of the manipulator are shown in Table 2.

Select the task space desired trajectory $\left\{\begin{array}{l}x_{d}=-0.25 \cos (\pi t) \mathrm{mm} \\ y_{d}=0.2-0.2 \cos ((\pi / 2) t) \mathrm{mm}\end{array}\right.$. The initial position is $\left[\begin{array}{ll}x_{d 0} & y_{d 0}\end{array}\right]=\left[\begin{array}{ll}-0.25 & 0\end{array}\right]^{T}$. Select the functions $d_{x}=\sin (\pi t)$ and $d_{y}=\sin (2 \pi t)$ for disturbance of both joints.

For the sake of implementing the tracking control in the joint space, based on the content described in Section 2.1, the 
TABLE 2: Simulation parameters of the two-link manipulator.

\begin{tabular}{lccc}
\hline$m_{1} / \mathrm{kg}$ & 0.8 & $\mathrm{~s}_{1} / \mathrm{m}$ & 0.125 \\
$m_{2} / \mathrm{kg}$ & 0.8 & $s_{2} / \mathrm{m}$ & 0.125 \\
$l_{1} / \mathrm{m}$ & 0.25 & $I_{1} /\left(\mathrm{kg} \cdot \mathrm{m}^{2}\right)$ & 0.05 \\
$l_{2} / \mathrm{m}$ & 0.25 & $I_{2} /\left(\mathrm{kg} \cdot \mathrm{m}^{2}\right)$ & 0.05 \\
$\mathrm{~g} /\left(\mathrm{m} / \mathrm{s}^{2}\right)$ & 9.8 & & \\
\hline
\end{tabular}

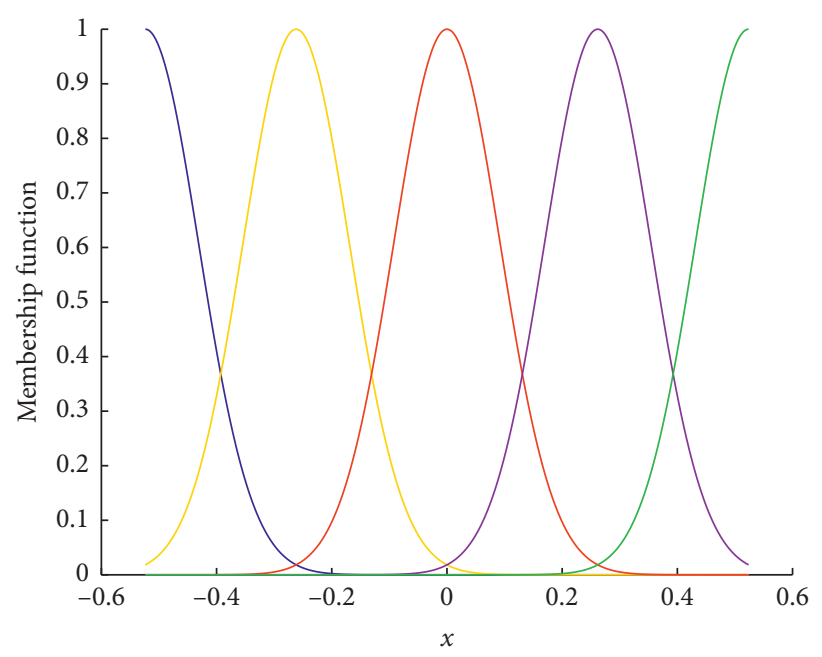

Figure 3: Membership function.

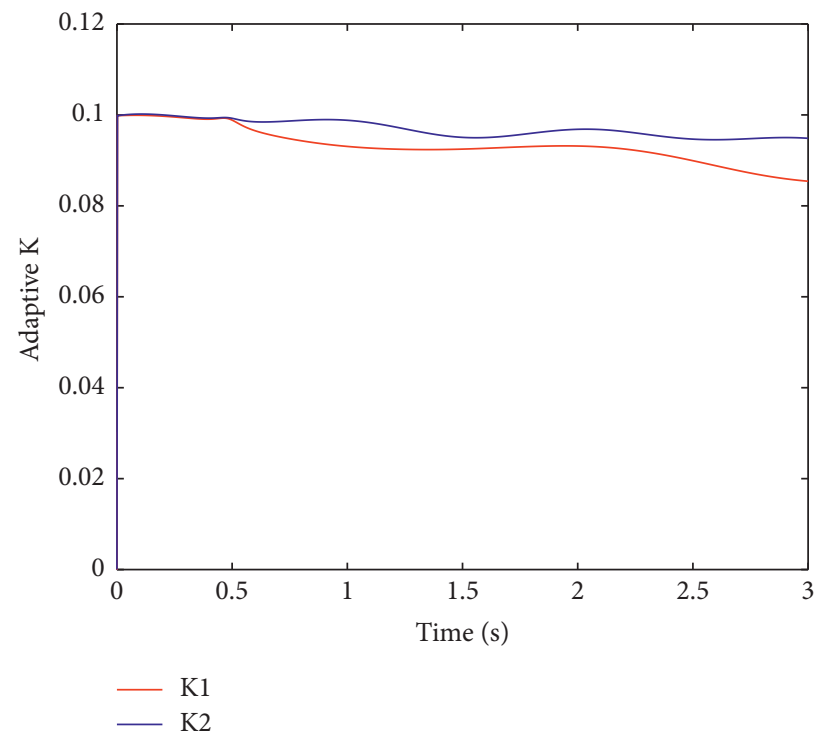

FIgURE 4: Adaptive gain variation of two joints.

expected trajectory and the initial joint angle of the joint are obtained by the inverse solution.

The sliding mode controller parameters are chosen as $\Lambda=\operatorname{diag}(10,10), A=\operatorname{diag}(150,150)$. The robust controller parameters are $\gamma=2$ and $\varepsilon=0.01$. Select the fuzzy membership function as shown in Figure 3.

First, conduct simulation by adopting the traditional SMC based on the fixed gain and SMC based on adaptive fuzzy gain adjustment so as to reflect the effect of switching gain adjustment by using fuzzy adaptive control on the improvement of sliding mode control buffeting. The adaptive gain changes of the two joints are shown in Figure 4.

In order to better compare the effect of sliding mode adaptive gain and fixed gain, for fixed gain, we adopt three different $K$ values $(K=2 ; K=15 ; K=100)$. The sliding mode control input signal chattering phenomenon of two joints with three different $K$ values is shown in Figures 5 and 6 . Figures 7 and 8 show the sliding mode control input signal with adaptive gain. 

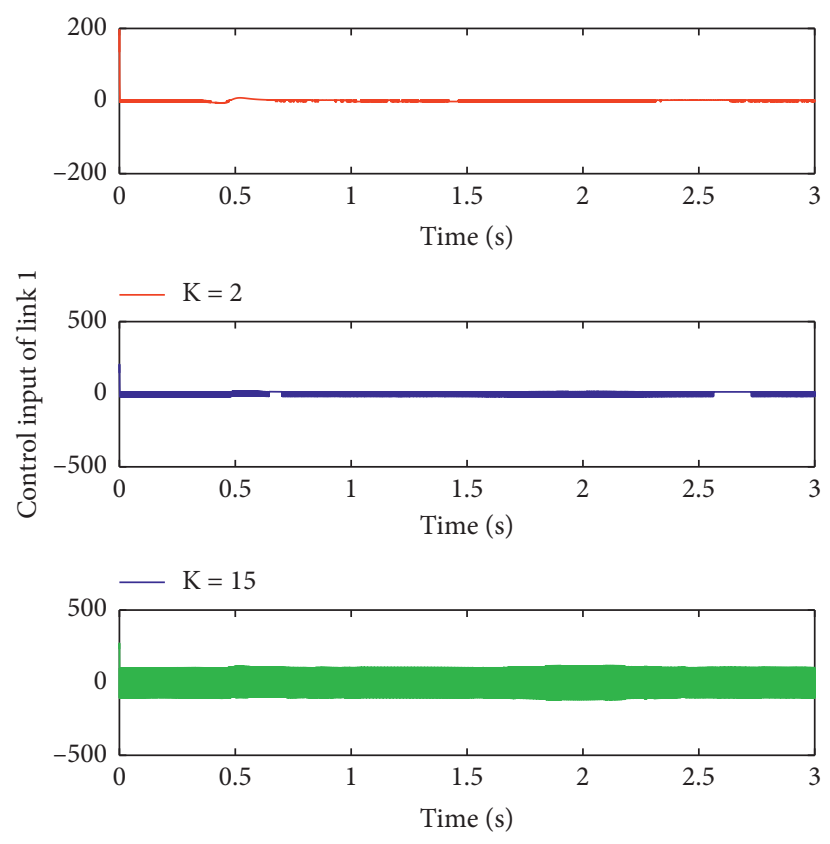

— $\mathrm{K}=100$

FIgURE 5: Control input of link 1 (fixed gain).
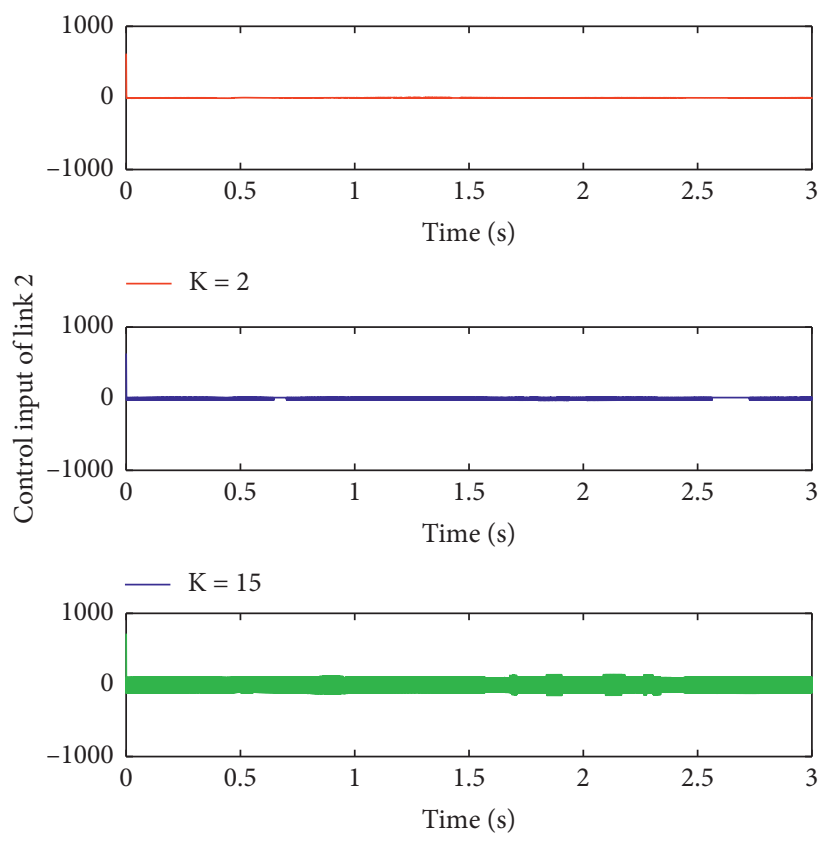

- K $=100$

Figure 6: Control input of link 2 (fixed gain).

From the figures, we can see that the SMC input signal with fixed gain has obvious chattering, and the larger the $\mathrm{K}$, the more obvious the chattering, while adoption of SMC based on fuzzy adaptive gain adjustment can effectively improve the chattering phenomenon and keep the input signal smooth.
The adaptive fuzzy sliding mode robust controller proposed in this paper is used for trajectory tracking of the two-joint manipulator. The simulation results are demonstrated in Figures 9-14. Among them, Figure 9 shows the position tracking of both joints. Figure 10 displays the tracking error of both joints. Figure 11 shows the velocity 


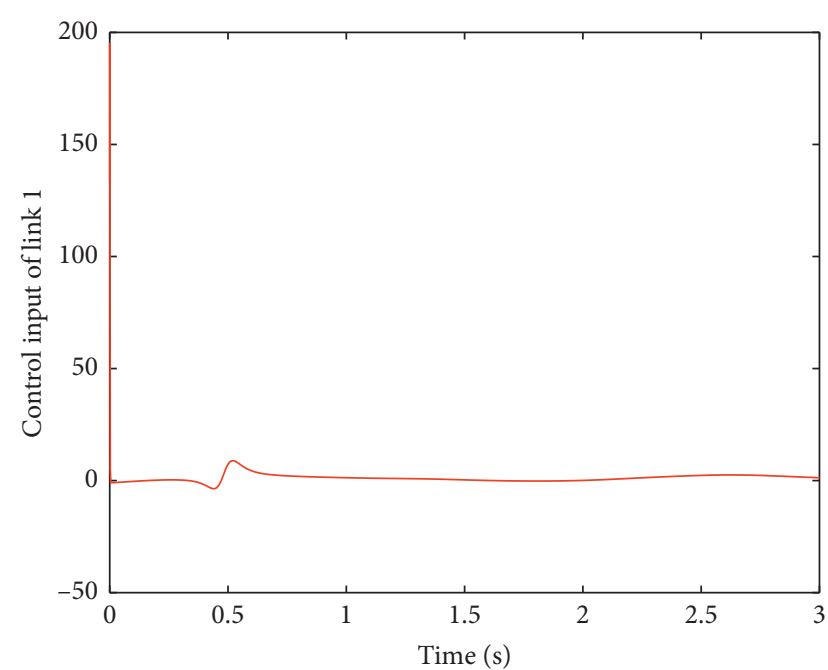

FIgURe 7: Control input of link 1 (adaptive gain).

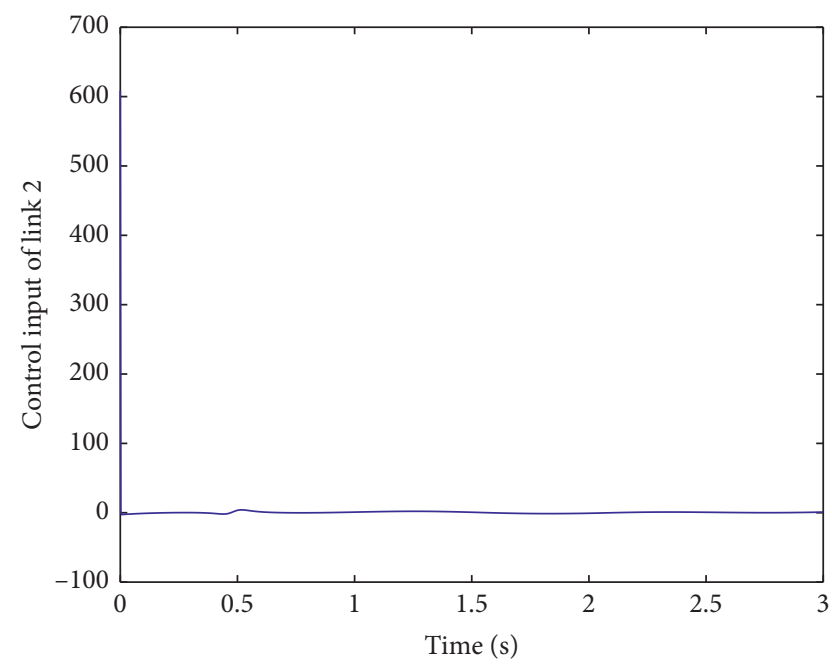

FIgURE 8: Control input of link 2 (adaptive gain).
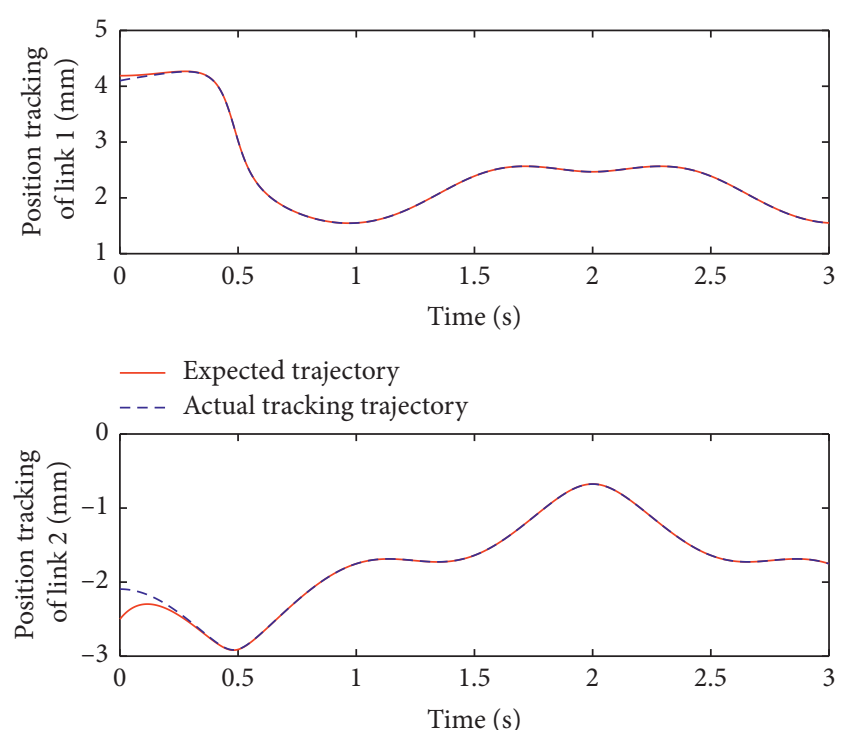

- Expected trajectory

- - - Actual tracking trajectory

Figure 9: Position tracking of two joints.
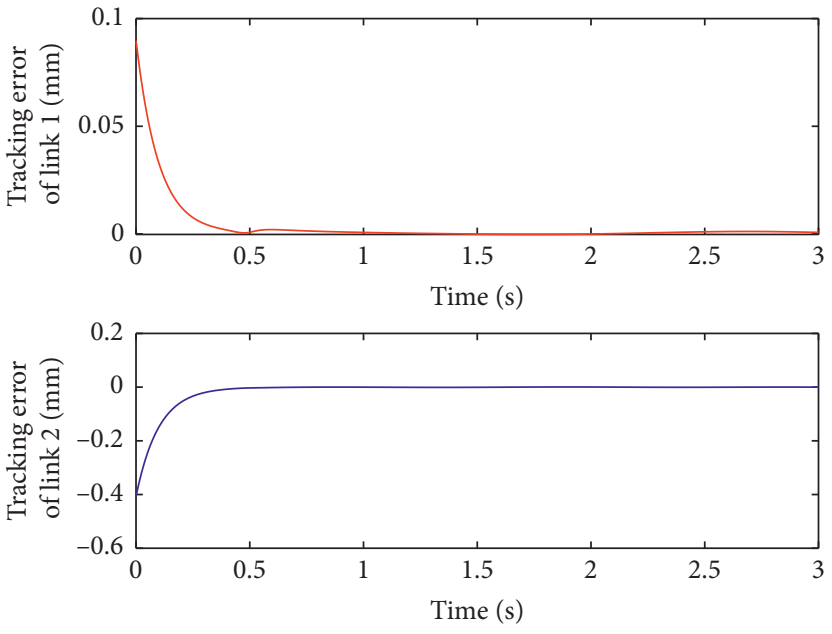

Figure 10: Position error of two joints.

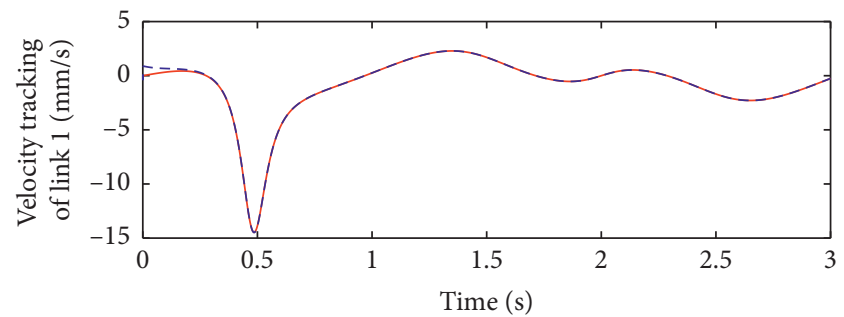

— Expected velocity

- _ Actual velocity tracking

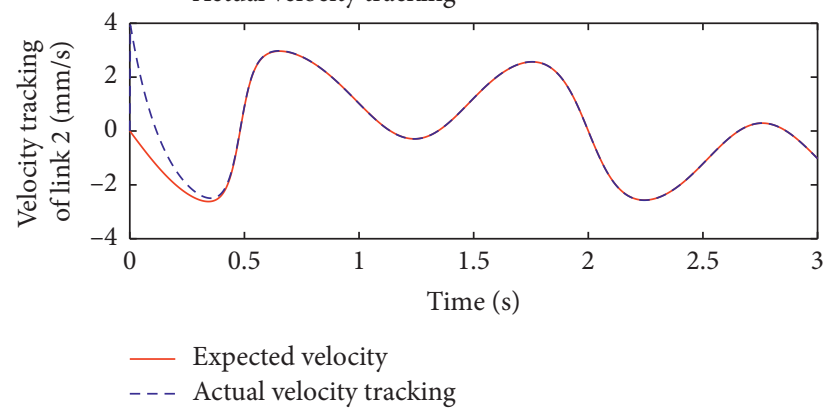

FIGURE 11: Velocity tracking of two joints.
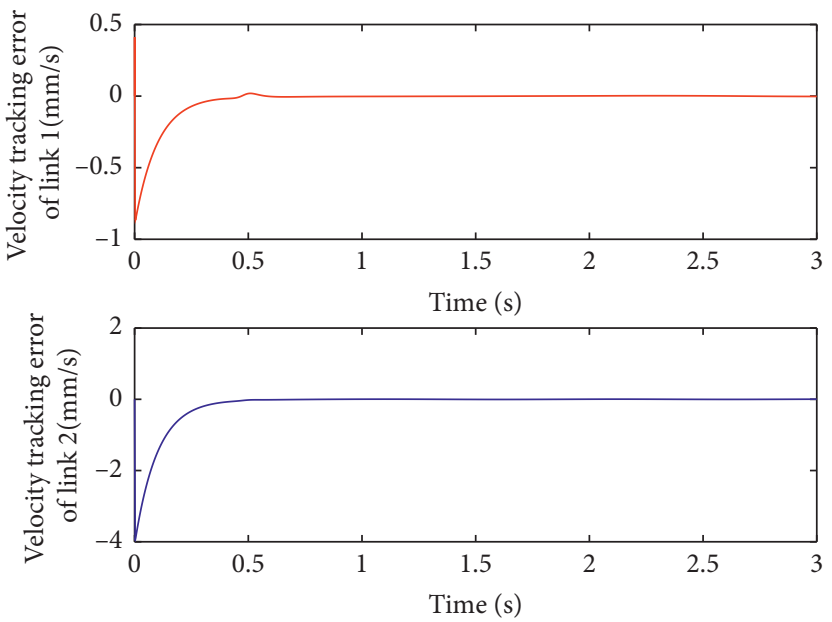

FIGURE 12: Velocity error of two joints. 


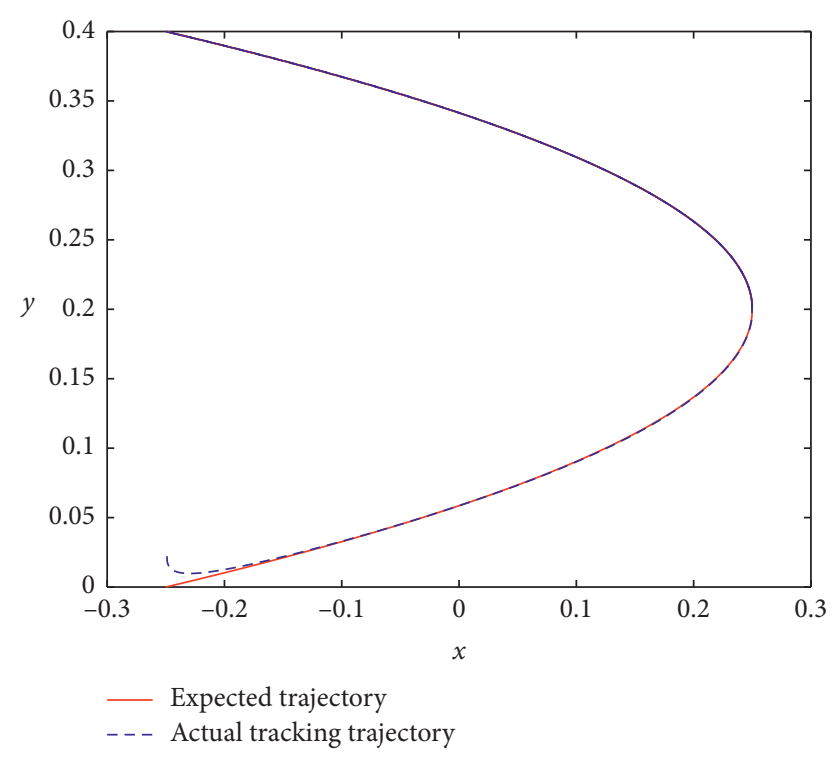

FIgURE 13: Trajectory tracking in the task space.

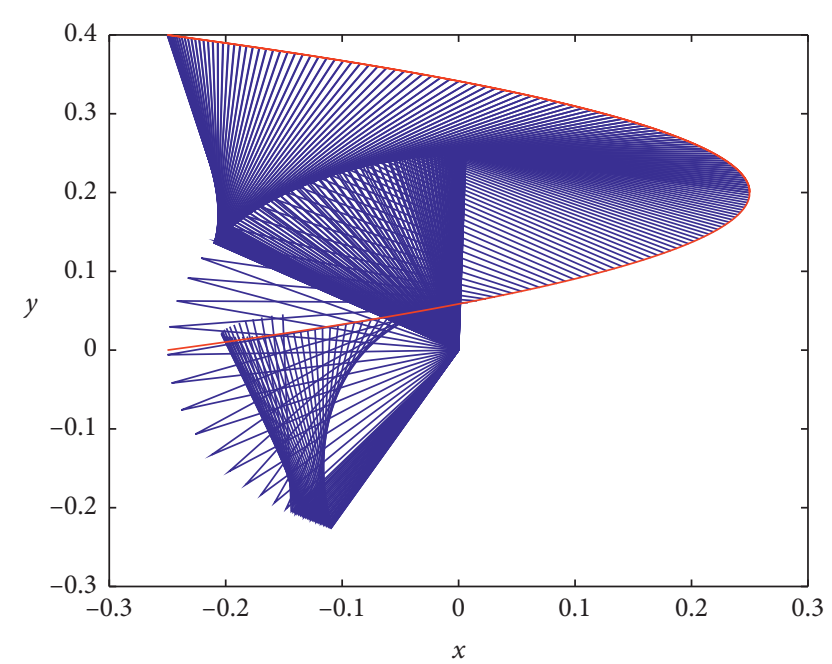

Figure 14: Pose trajectory.

TABLE 3: Simulation error table.

\begin{tabular}{cccc}
\hline \multicolumn{2}{c}{ Axis } & Position error $(\mathrm{mm})$ & Velocity error $(\mathrm{mm})$ \\
\hline \multirow{4}{*}{$x$-axis } & Max & 0.0889 & 0.8656 \\
& Mean & 0.0036 & 0.0312 \\
& Rms & 0.0116 & 0.1146 \\
\multirow{3}{*}{$y$-axis } & Max & 0.0889 & 3.9522 \\
& Mean & 0.0036 & 0.1370 \\
& Rms & 0.0116 & 0.5227 \\
\hline
\end{tabular}

tracking of both joints. Figure 12 displays the velocity tracking error of both joints. Figure 13 displays the tracking trajectory at the terminal of the task space for the robotic manipulator. Figure 14 displays the pose trajectory of the manipulator.

It can be seen from the simulation results that the proposed robotic manipulator control method enjoys high trajectory tracking precision. In addition to the initial stage when there is a minor tracking error, it basically ensures high-precision tracking with tracking error being zero. Simulation error values of two axes are shown in Table 3.

\section{Conclusions}

This article proposes an adaptive fuzzy sliding mode robust trajectory tracking control algorithm for the robotic manipulator with uncertain parameters and external disturbance. The algorithm combines SMC tracking controller, switched gain adaptive fuzzy controller, and robust control algorithms to compensate for modeling error and disturbance, weakens the buffeting of SMC, and ensures high precision of robot trajectory tracking. The simulation results carried on a two-link robotic manipulator demonstrate the validity and superiority of the proposed method.

\section{Data Availability}

The data used to support the findings of this study are included within the article.

\section{Conflicts of Interest}

The authors declare that they have no conflicts of interest.

\section{Acknowledgments}

This research was supported by the Joint Fund of Zhejiang Provincial Natural Science Foundation (Grant no. LTY20E050001).

\section{References}

[1] N. Yagiz, Y. Hacioglu, and Y. Z. Arslan, "Load transportation by dual arm robot using sliding mode control," Journal of Mechanical Science and Technology, vol. 24, no. 5, pp. 11771184, 2010.

[2] S. Wang, L. Tao, Q. Chen, J. Na, and X. Ren, "USDE-based sliding mode control for servo mechanisms with unknown system dynamics," IEEE/ASME Transactions on Mechatronics, vol. 25, no. 2, pp. 1056-1066, 2020.

[3] R. Cui, L. Chen, C. Yang, and M. Chen, "Extended state observer-based integral sliding mode control for an underwater robot with unknown disturbances and uncertain nonlinearities," IEEE Transactions on Industrial Electronics, vol. 64, no. 8, pp. 6785-6795, 2017.

[4] Q. Chen, S. Xie, M. Sun, and X. He, "Adaptive nonsingular fixed-time attitude stabilization of uncertain spacecraft," IEEE Transactions on Aerospace and Electronic Systems, vol. 54, no. 6, pp. 2937-2950, 2018.

[5] M. Tao, Q. Chen, X. He, and M. Sun, "Adaptive fixed-time fault-tolerant control for rigid spacecraft using a double power reaching law," International Journal of Robust and Nonlinear Control, vol. 29, no. 12, pp. 4022-4040, 2019.

[6] J. Na, B. Wang, G. Li, S. Zhan, and W. He, "Nonlinear constrained optimal control of wave energy converters with adaptive dynamic programming," IEEE Transactions on Industrial Electronics, vol. 66, no. 10, pp. 7904-7915, 2019. 
[7] H. S. Kim, Y. M. Cho, and K.-I. Lee, "Robust nonlinear task space control for 6 DOF parallel manipulator," Automatica, vol. 41, no. 9, pp. 1591-1600, 2005.

[8] V. T. Yen, W. Y. Nan, P. Van Cuong, N. X. Quynh, and V. H. Thich, "Robust adaptive sliding mode control for industrial robot manipulator using fuzzy wavelet neural networks," International Journal of Control, Automation and Systems, vol. 15, no. 6, pp. 2930-2941, 2017.

[9] Q. Chen, H. Shi, and M. Sun, "Echo state network-based backstepping adaptive iterative learning control for strictfeedback systems: an error-tracking approach," IEEE Transactions on Cybernetics, vol. 50, no. 7, pp. 3009-3022, 2020.

[10] J. J. Slotine and W. Li, Applied Nonlinear Control, PrenticeHall, Englewood Cliffs, NJ, USA, 1991.

[11] N. Kapoor and J. Ohri, "Fuzzy sliding mode controller (FSMC) with global stabilization and saturation function for tracking control of a robotic manipulator," Journal of Control and Systems Engineering, vol. 1, no. 2, pp. 50-56, 2013.

[12] L. Hsu, "Smooth sliding control of uncertain systems based on a prediction error," International Journal of Robust and Nonlinear Control, vol. 7, no. 4, pp. 353-372, 1997.

[13] V. Acary, B. Brogliato, and Y. V. Orlov, "Chattering-free digital sliding-mode control with state observer and disturbance rejection," IEEE Transactions on Automatic Control, vol. 57, no. 5, pp. 1087-1101, 2012.

[14] Y. Fan, K. Xing, and X. Jiang, "Fuzzy adaptation algorithms' control for robot manipulators with uncertainty modelling errors," Complexity, vol. 2018, pp. 1-8, 2018.

[15] J. Na, Y. Huang, X. Wu, S.-F. Su, and G. Li, “Adaptive finitetime fuzzy control of nonlinear active suspension systems with input delay," IEEE Transactions on Cybernetics, vol. 50, no. 6, pp. 2639-2650, 2019.

[16] T.-F. Lee and A.-C. Huang, "Vibration suppression in beltdriven servo systems containing uncertain nonlinear dynamics," Journal of Sound and Vibration, vol. 330, no. 1, pp. 17-26, 2011.

[17] E. Kim and S. Lee, "Output feedback tracking control of MIMO systems using a fuzzy disturbance observer and its application to the speed control of a PM synchronous motor," IEEE Transactions on Fuzzy Systems, vol. 13, no. 6, pp. 725741, 2005.

[18] S. Wang and J. Na, "Parameter estimation and adaptive control for servo mechanisms with friction compensation," IEEE Transactions on Industrial Informatics, p. 1, 2019.

[19] T. L. Shen, Fundamentals of Robust Control for Robots, Tsinghua university press, Beijing, China, 2000.

[20] M. Veysi, M. R. Soltanpour, and M. H. Khooban, "A novel self-adaptive modified bat fuzzy sliding mode control of robot manipulator in presence of uncertainties in task space," Robotica, vol. 33, no. 10, pp. 2045-2064, 2015.

[21] Y.-C. Liu and N. Chopra, "Controlled synchronization of heterogeneous robotic manipulators in the task space," IEEE Transactions on Robotics, vol. 28, no. 1, pp. 268-275, 2012. 